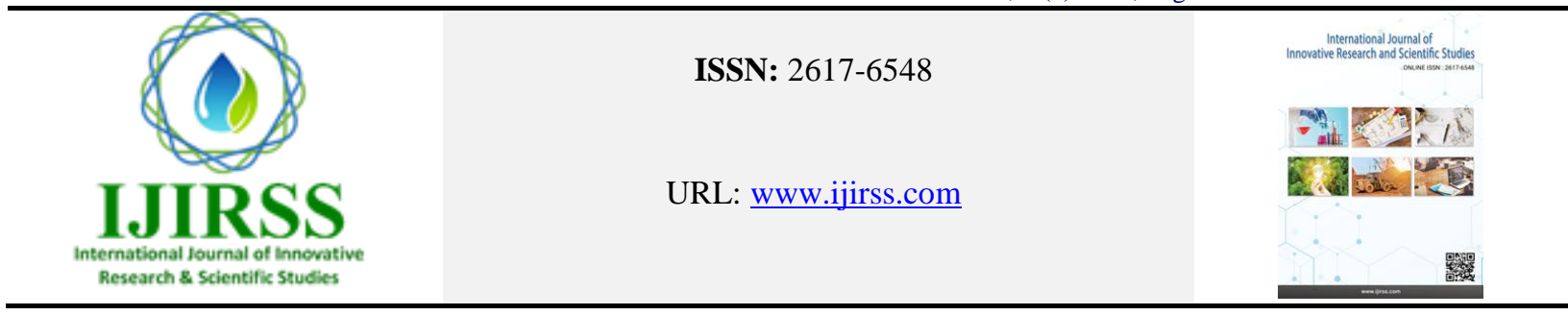

\title{
A Review on Production of Biodiesel from Waste Cooking Oils
}

\author{
Muzhda Azizi ${ }^{1 *}$, Sweeta Akbari ${ }^{2}$ \\ ${ }^{1}$ Faculty of Chemical Engineering Technology, Jawzjan University, Jawzjan, Afghanistan \\ ${ }^{2}$ Faculty of Chemical \& Natural Resources Engineering, Universiti Malaysia Pahang, Lebuhraya Tun Razak, 26300 Gambang, Pahang, \\ Malaysia \\ *Corresponding author: Muzhda Azizi (muzhdaazizi7@gmail.com)
}

\section{Graphical Abstract}

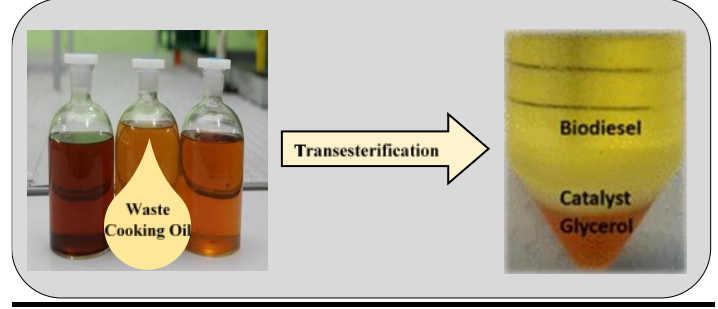

\begin{abstract}
Nowadays, preservation of natural resources on earth is one of the most important concerns of humanity. In this regard, increasing the consumption of energy is one of the most critical challenges that humans are facing. Because, on the one hand, the untapped use of different sources of energy from fossil fuels can destroy this natural resource and, on the other hand, pollution from the use of these resources is a serious threat to the environment. Recent research suggests that affordable, sustainable and environmentally friendly fuels, which can be a good alternative to fossil fuels, have become more important. Therefore, biodiesel has made it possible to release less greenhouse gas emission and low toxicity emissions, which can partly meet fuel requirements and is the best alternative for petroleum diesel. In addition, the waste cooking oils are a major source of biodiesel for their essential compounds, such as glycerol. The use of waste cooking oils can reduce biodiesel production cost by 60 to 90 percent. Therefore, the main objective of this review is to study the production of biodiesel using transesterification reaction of waste cooking oil as an alternative fuel to petroleum diesel that can be used easily in diesel engines.
\end{abstract}

Keywords: Biodiesel, waste cooking oil, transesterification, methanol, glycerol, catalyst.

DOI: 10.53894 /ijirss.v1i2.8

Funding: This study received no specific financial support.

History: Received: 17 August 2018/Revised: 10 September 2018/Accepted: 25 September 2018/Published: 15 October 2018

Licensed: This work is licensed under a Creative Commons Attribution 4.0 License (c) $)_{\mathrm{EY}}$

Acknowledgement: Both authors contributed to the conception and design of the study.

Competing Interests: The authors declare that they have no conflict of interests.

Transparency: The authors confirm that the manuscript is an honest, accurate, and transparent account of the study was reported; that no vital features of the study have been omitted; and that any discrepancies from the study as planned have been explained.

Ethical: This study follows all ethical practices during writing. 


\title{
مرورى بر توليد بيوديزل از روغن هاى باقيمانده بخت و بز
}

\author{
مزده عزيزى، سويتا اكبرى \\ افكولته /نجنيرى تكنالوزى كبياوى، بوهنتون جوزجان، جوزجان، افغانستان

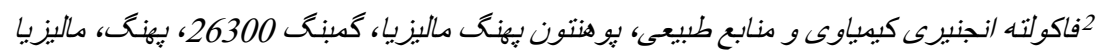

خلاصه كر افيكى

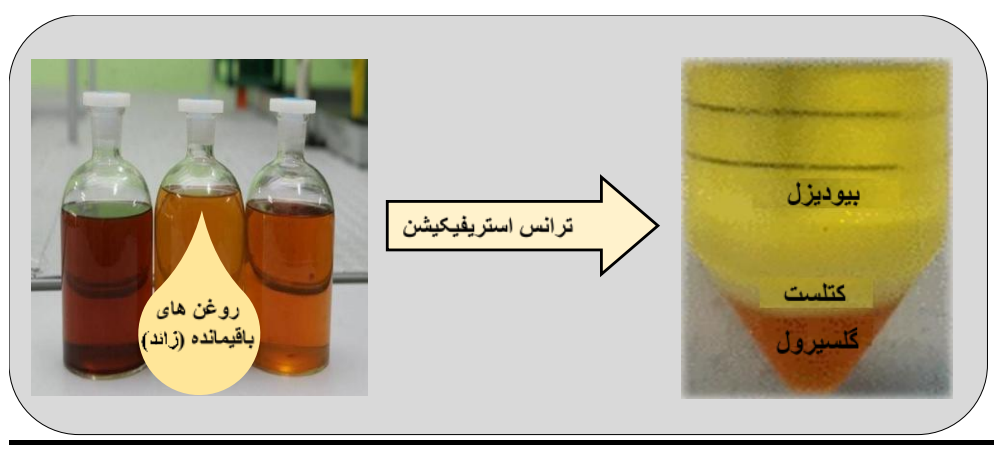

\section{خلاصه}

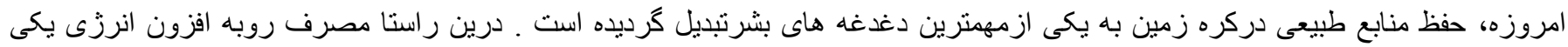

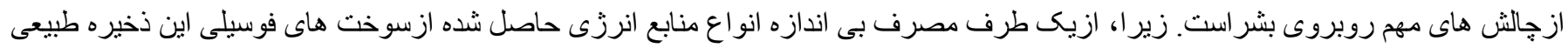

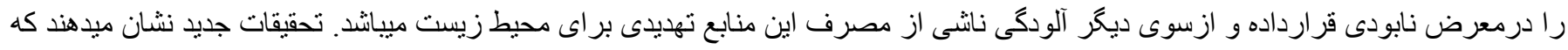

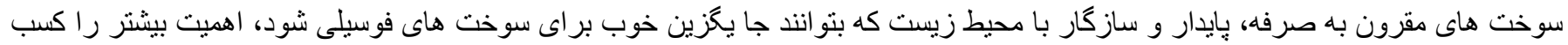

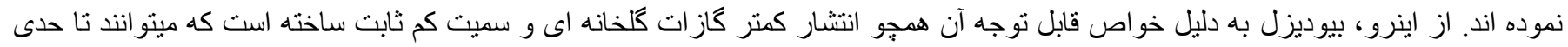

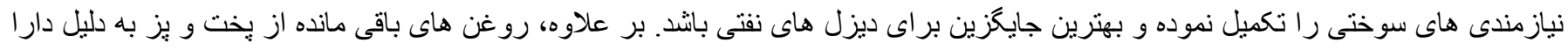

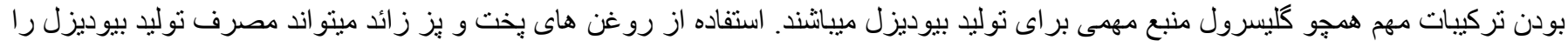

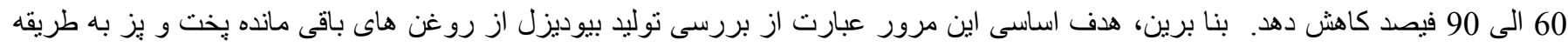

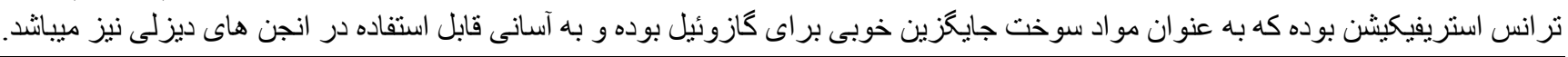

كلمات كليبى: بيوديزل، روغن هاى باقيمانده، كليسرول، تر انس استريفيكيشن، ميتانول، كتلست

1

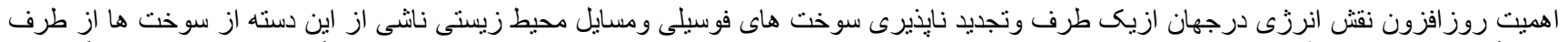

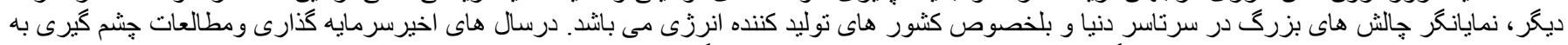

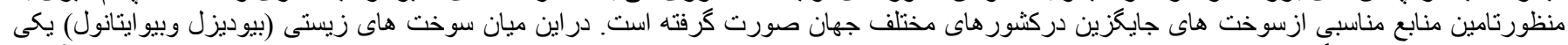

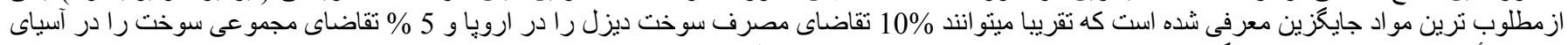

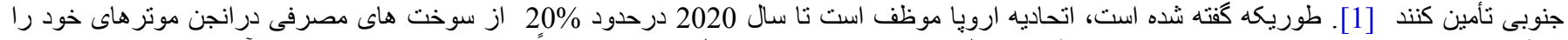

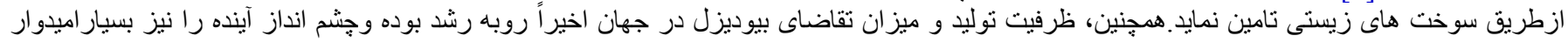

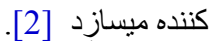

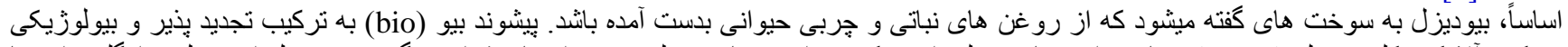

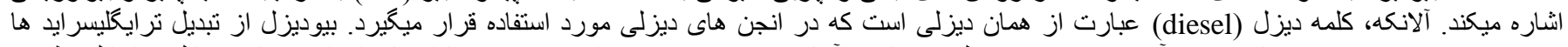
(triglycerides)

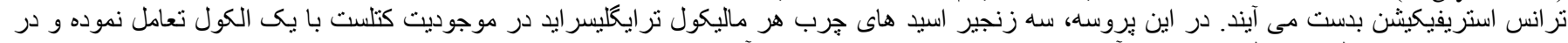

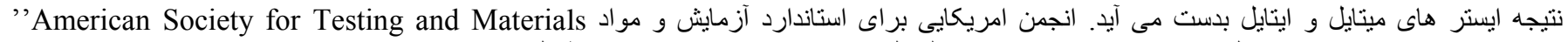

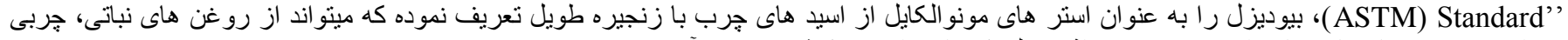

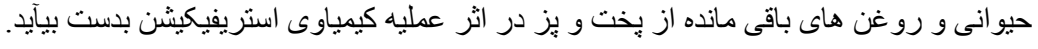




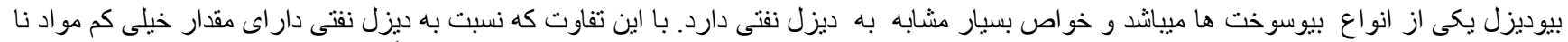

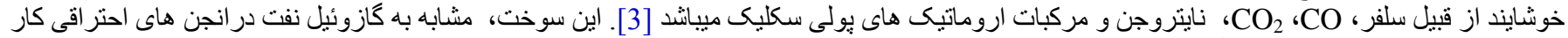

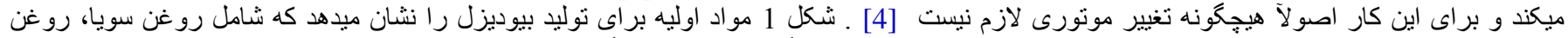

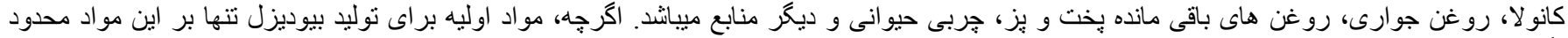

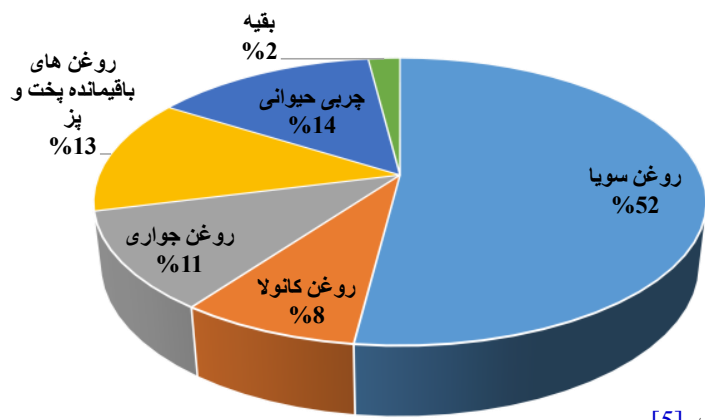

شكل 1. مواد اوليه براى توليد بيوديزل [5] .

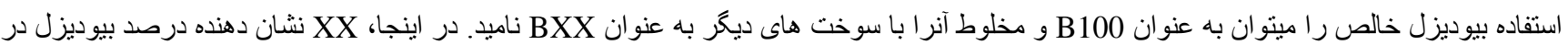

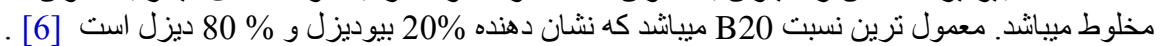

بر اساس تحقيقات بعمل آمده آلمان، آمريكاو ماليزيا از جمله كثور هايى هستند كه روى ائن مواد ادئ سوخت كار كرده اند، اين محروق در آمريكا بعنوان يكى

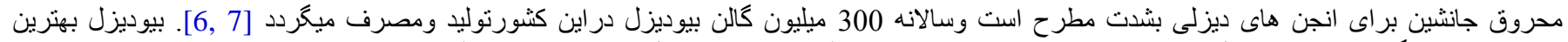

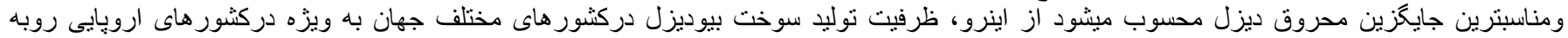

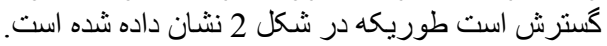

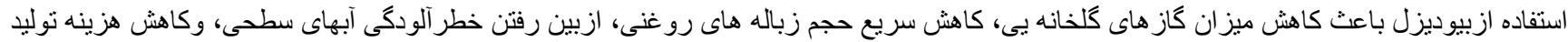

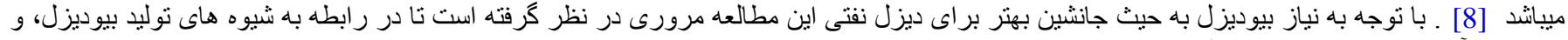

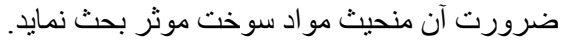

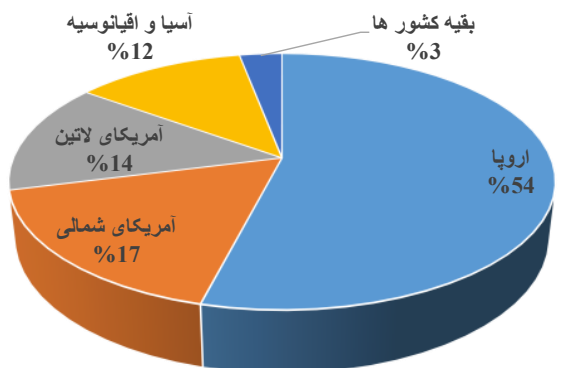

شكل 2. بيش بينى باز ار جهانى بيوديزل تا سال 2027 ميلادى [9] .

روش هاى توليخ بيوديزل با استفاده از روغن هاى باقيمانده

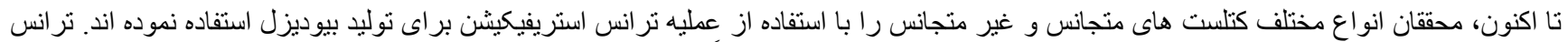

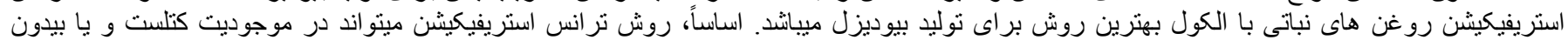

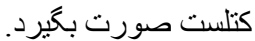

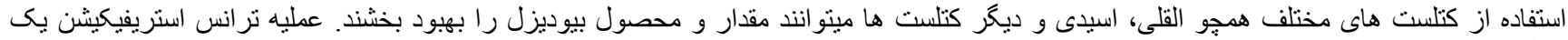

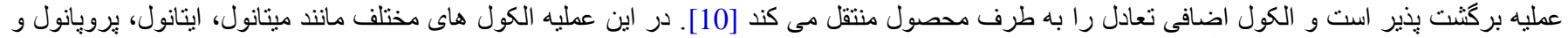

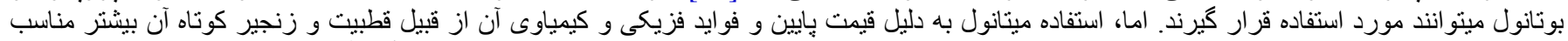

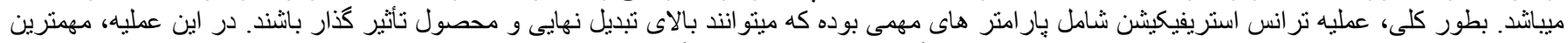

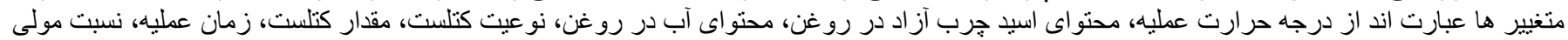

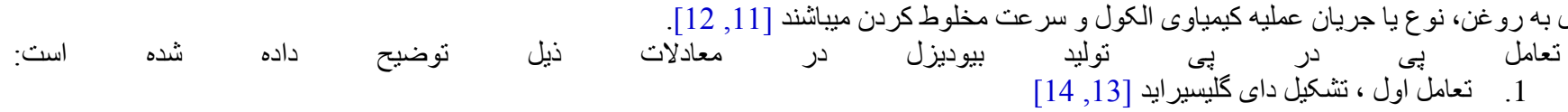




$$
\begin{aligned}
& \mathrm{C}_{1} \mathrm{H}_{2}-\mathrm{OOR}_{1} \quad \mathrm{C}_{1} \mathrm{H}_{2}-\mathrm{OH}
\end{aligned}
$$

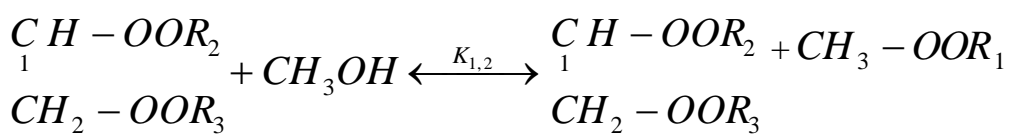

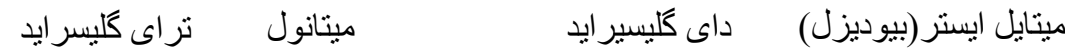

2. 2 - ت تعامل دوم، تثكيل مونوكليسير ايد

$$
\begin{aligned}
& \underset{1}{\mathrm{CH}_{2}}-\mathrm{OH} \quad \mathrm{C}_{1} \mathrm{H}_{2}-\mathrm{OH} \\
& \underset{1}{\mathrm{C}} \mathrm{H}-\mathrm{OOR}_{2}+\mathrm{CH}_{3} \mathrm{OH} \stackrel{\mathrm{K}_{3,4}}{\longleftrightarrow} \underset{1}{\mathrm{C} H}-\mathrm{OOR}_{2}+\mathrm{CH}_{3}-\mathrm{OOR}_{2} \\
& \mathrm{CH}_{2}-\mathrm{OOR}_{3} \mathrm{CH}_{2}-\mathrm{OH}
\end{aligned}
$$

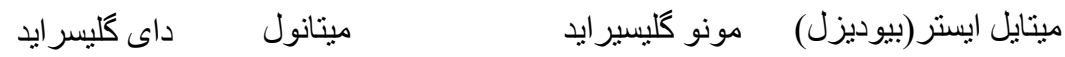$$
\text { 3. تعامل سوم، تثكيل كليسيرول و ايسترنهايى (بيوديزل) }
$$

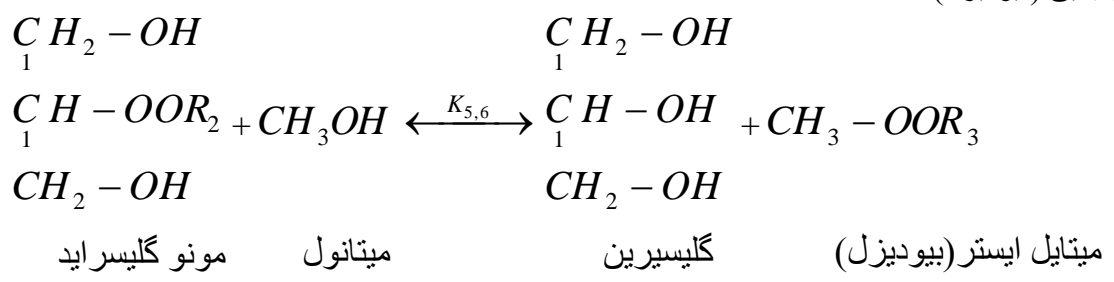

و يا بطور مختصر و سريع، اين عمليه با اساس تعامل يك مول تر ايكليسر ايد با سه مول ميتانول بوده كه در نتيجه باعث تنكيل سه مول ميتايل ايستر (بيوديزل) و يكى مول كليسرين ميكردد.

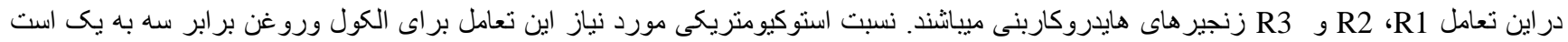

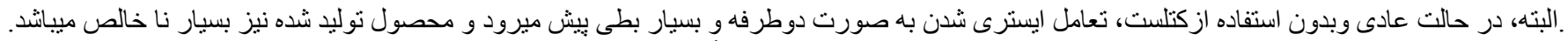

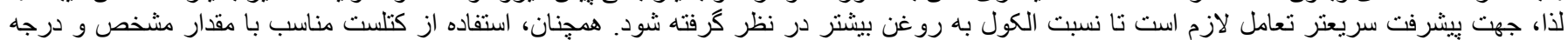

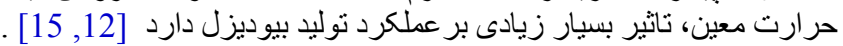

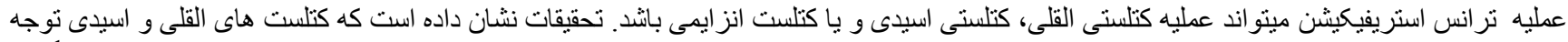

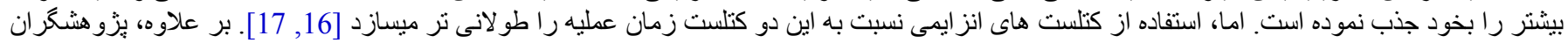

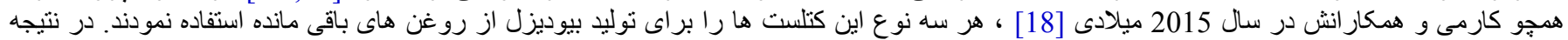

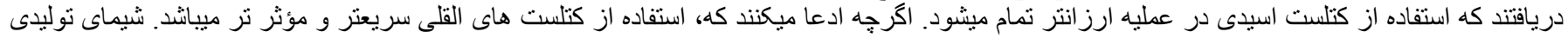

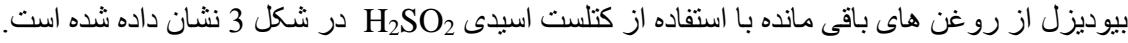

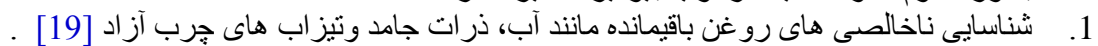

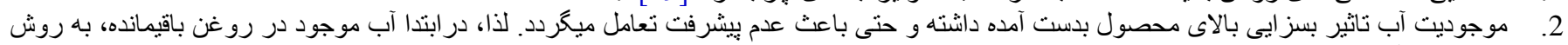

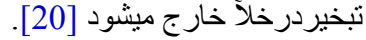

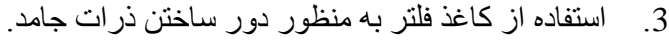

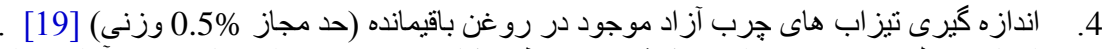

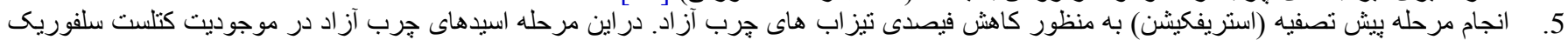

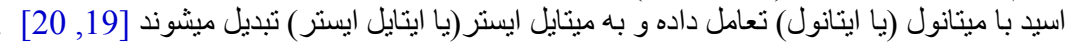

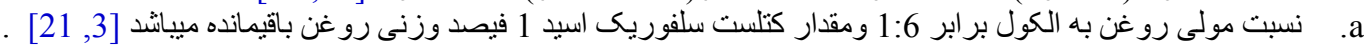

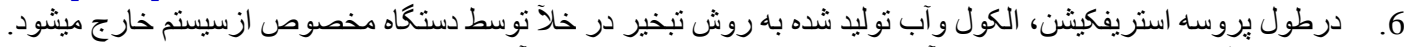

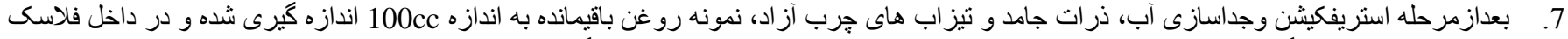

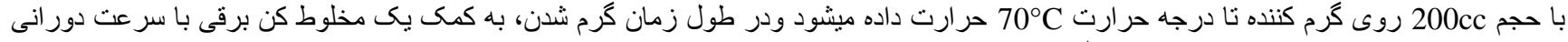

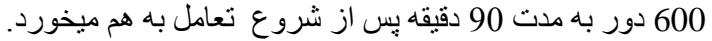

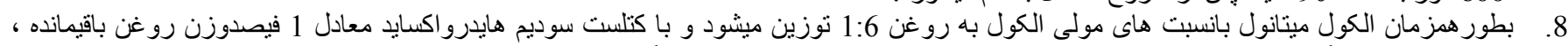
درداخل فلاسك ديكرى باحجم 100cc درحر ارت 


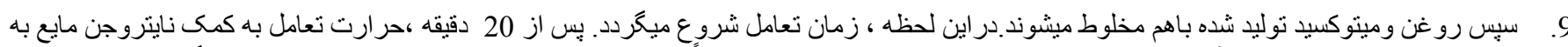

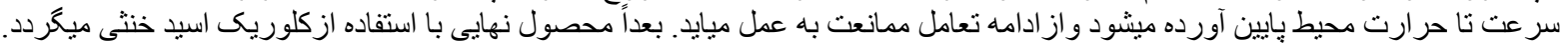

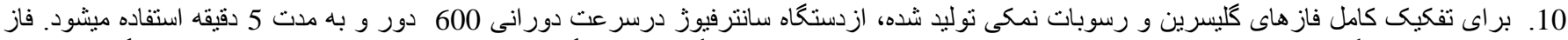

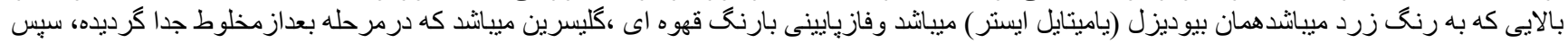

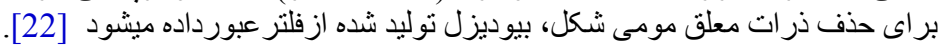

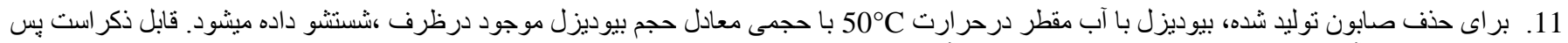

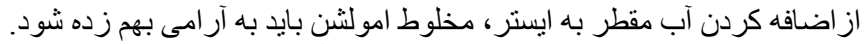

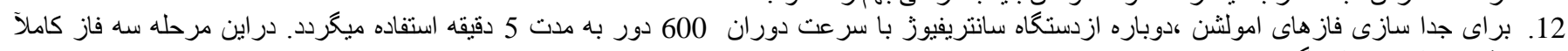

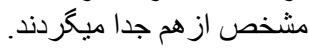

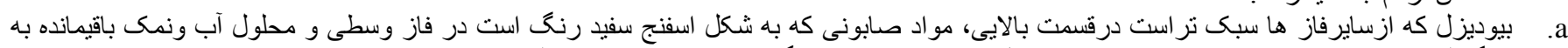

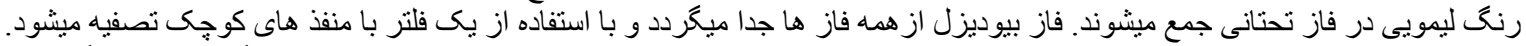

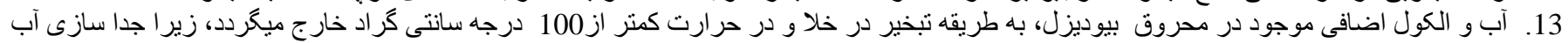

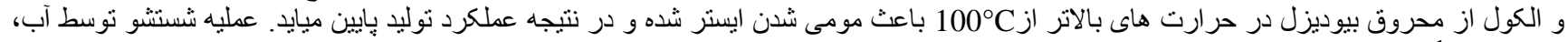

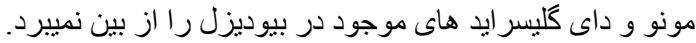

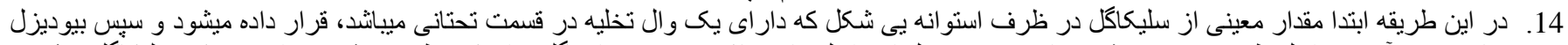

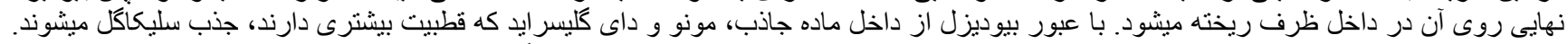

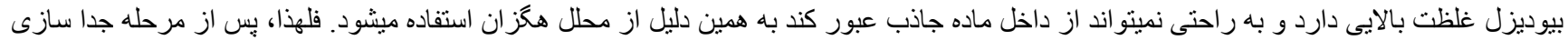

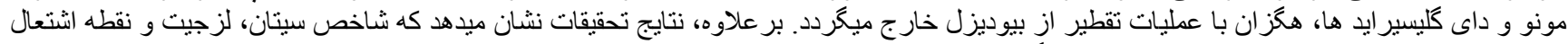

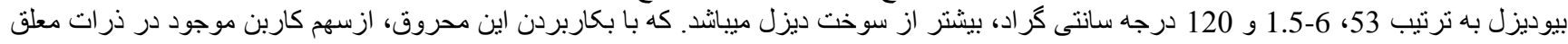

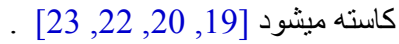

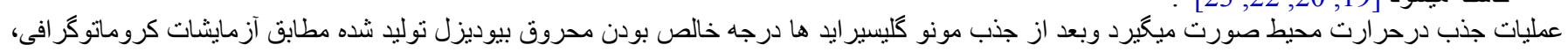
به بيش از 98.5 فيصد ميرسد.

\begin{tabular}{|c|c|}
\hline 1: & حرارت تعامل \\
\hline 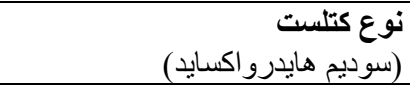 & $\begin{array}{r}\text { نوع الكول } \\
\text { (ميتانول) }\end{array}$ \\
\hline 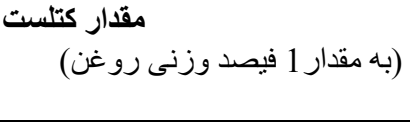 & شور ) شخلو مخلوط كردن ميخانيكى با 600 \\
\hline
\end{tabular}

5. ت اثر مدت اتجام تعامل بر عملكرد توليا بيوديزل

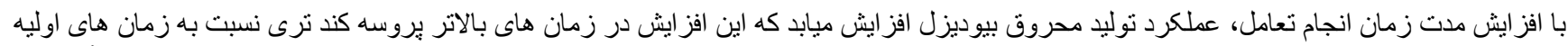

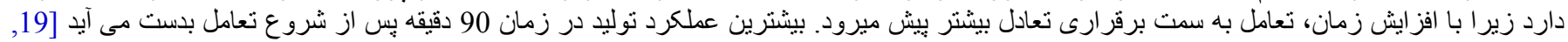

6. اثر نوع الكول بر عملكرد توليد بيوديزل

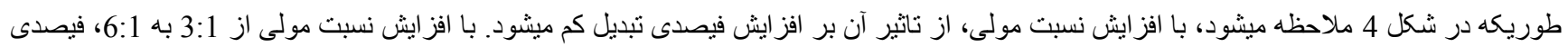

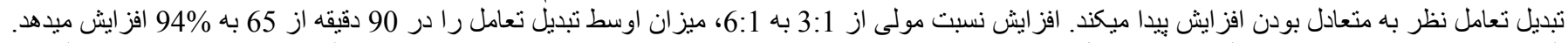

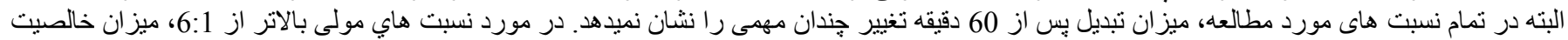

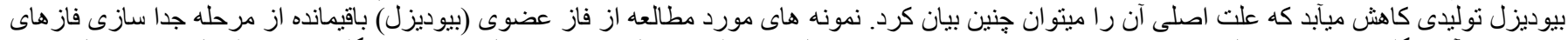

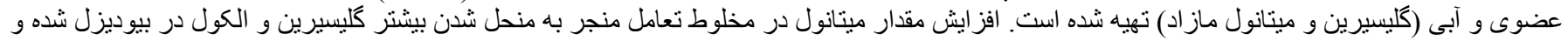

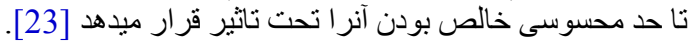




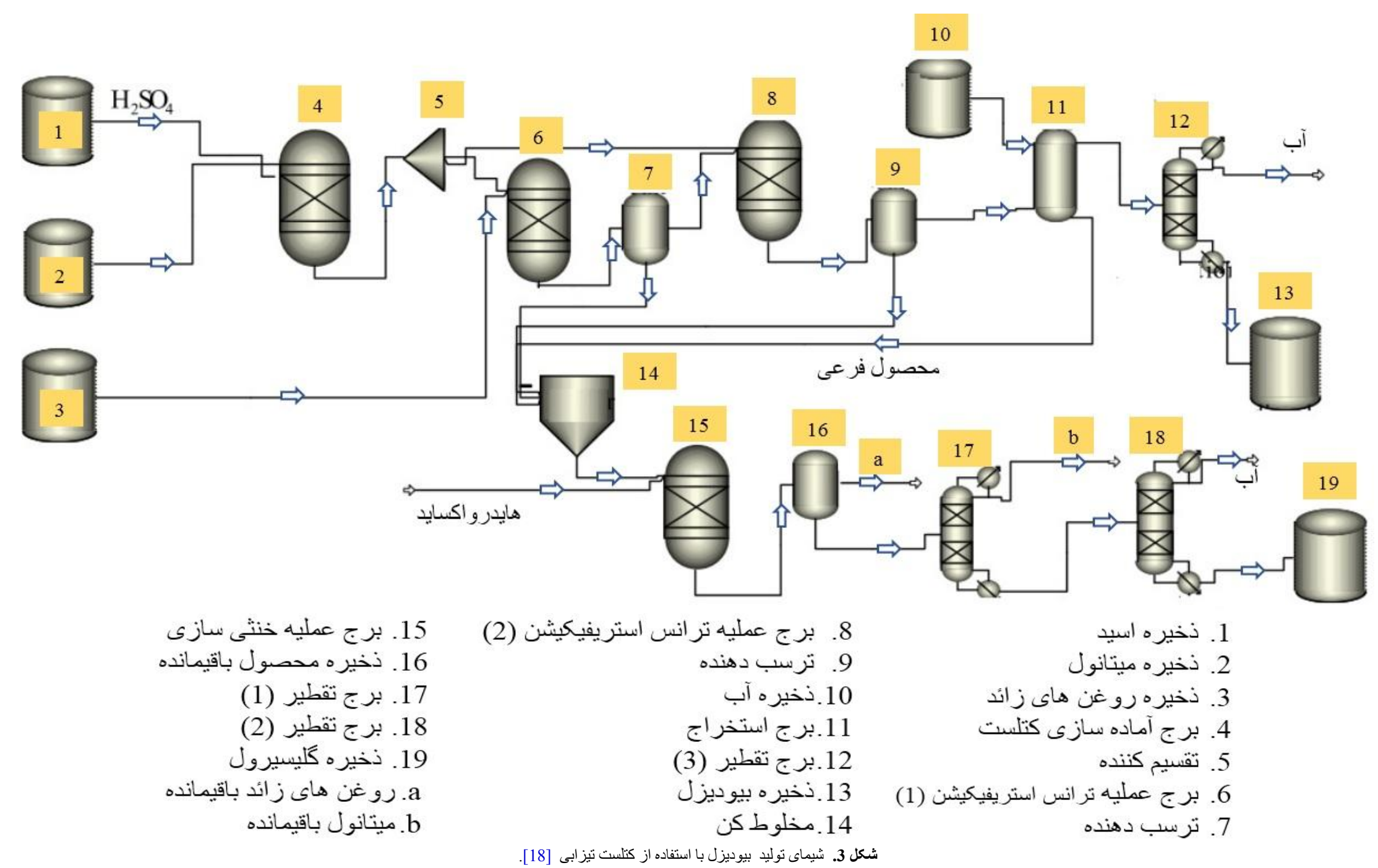




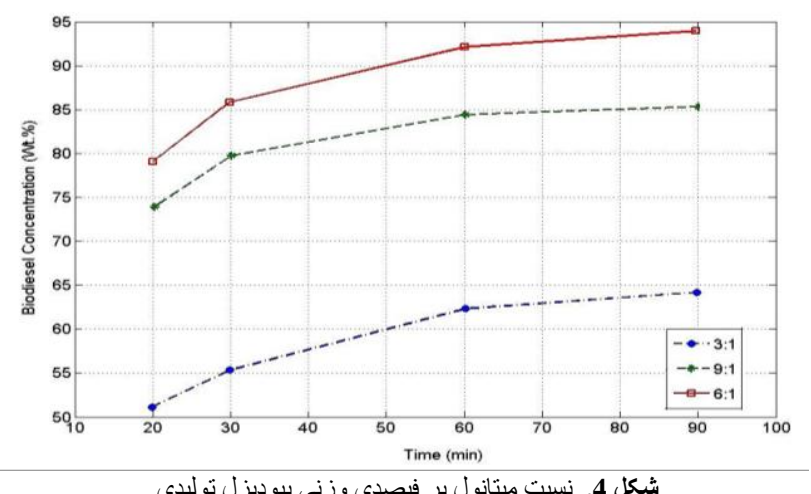

7. اثر درجه حرارت بر فيصدى تبديل تعامل

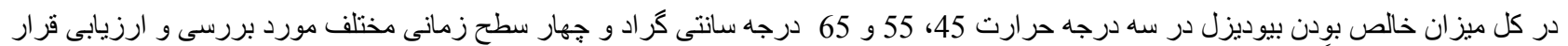

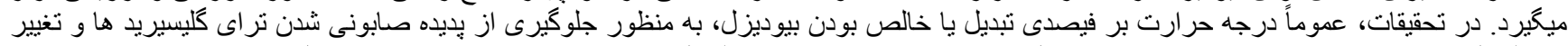

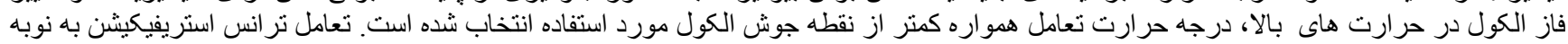

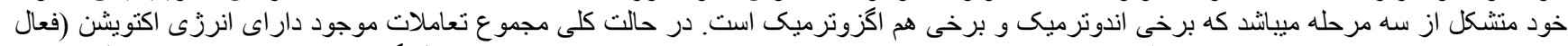

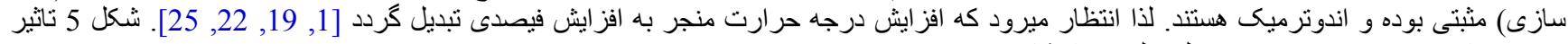

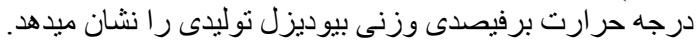

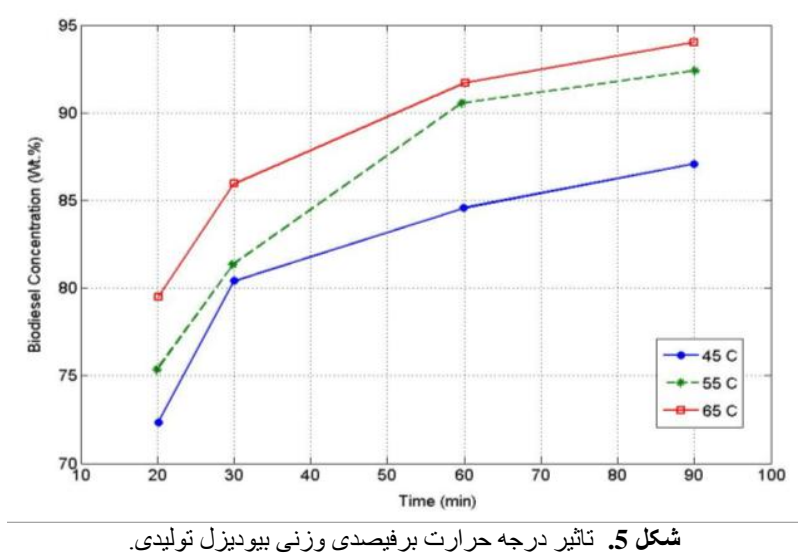

8.

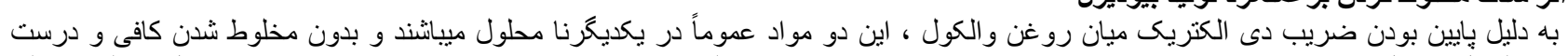

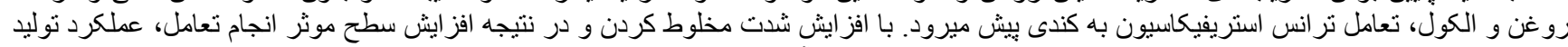

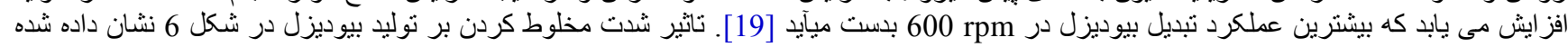

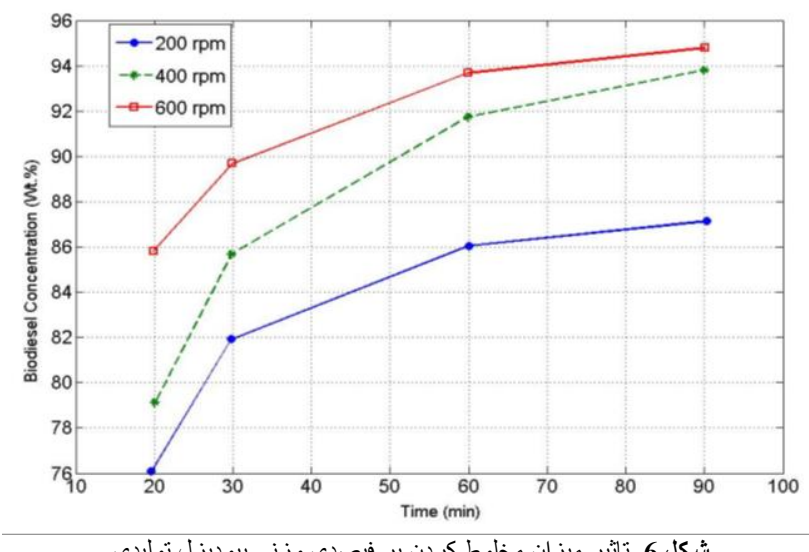

شكل 6. تاثير ميزان مخلوط كردن بر فيصدى وزنى بيوديزل توليدى. 


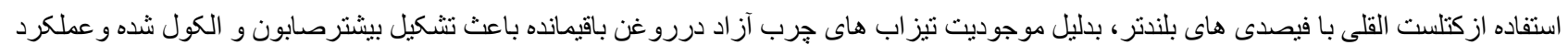

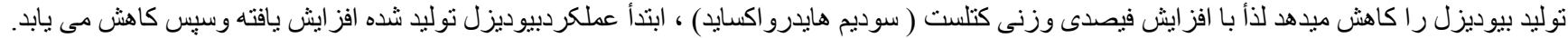

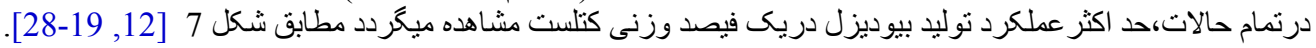

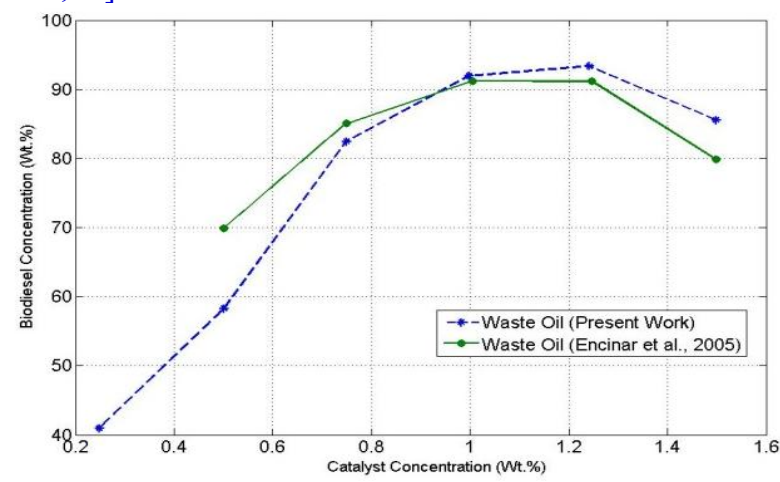

شكل 7. تاثير ميزان كتلست مورد استفاده برفيصدى وزنى بيوديزل توليدى.

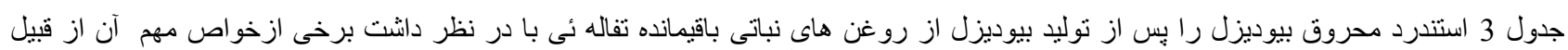

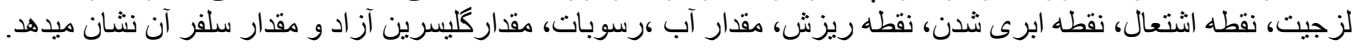

\begin{tabular}{|c|c|c|c|c|c|}
\hline \multirow[b]{2}{*}{ خصوصيت } & \multirow[b]{2}{*}{ واحد } & \multirow{2}{*}{ ASTM } & \multicolumn{2}{|c|}{ بيوديزل محدوه استاندرد محروق } & \multirow[b]{2}{*}{ نفازوئيل } \\
\hline & & & Min & Max & \\
\hline غلظت & $\mathrm{gr} / \mathrm{cm} 3$ & $\begin{array}{l}\text { ASTM } \\
\text { D4052 }\end{array}$ & 0.86 & 0.9 & 0.815 \\
\hline لزينيت & $\mathrm{Mm} 2 / \mathrm{s}$ & $\begin{array}{l}\text { ASTM } \\
\text { D4052 }\end{array}$ & 1.5 & 6 & 2.45 \\
\hline نقطنه & ${ }^{\circ} \mathrm{C}$ & $\begin{array}{l}\text { ASTM } \\
\text { D4052 }\end{array}$ & 120 & - & 61 \\
\hline شقدن ابرى & ${ }^{\circ} \mathrm{C}$ & $\begin{array}{l}\text { ASTM } \\
\text { D4052 } \\
\end{array}$ & -7 & - & -4 \\
\hline ريزش & ${ }^{\circ} \mathrm{C}$ & $\begin{array}{l}\text { ASTM } \\
\text { D4052 }\end{array}$ & -10 & - & -24 \\
\hline كليسرين & $\%$ vol & $\begin{array}{l}\text { ASTM } \\
\text { D4052 }\end{array}$ & 0.02 & & - \\
\hline رآبوبات & $\mathrm{g} / \mathrm{cm}^{3}$ & $\begin{array}{l}\text { ASTM } \\
\text { D4052 } \\
\end{array}$ & 0.05 & - & - \\
\hline مقدار سلفر & $\%$ & $\begin{array}{l}\text { D } 5453 \\
\text { ASTM }\end{array}$ & - & - & 2 \\
\hline
\end{tabular}

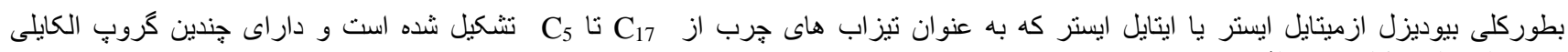
بازنجير هاى هايدروكاربنى ميباشند.

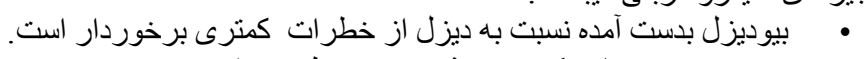

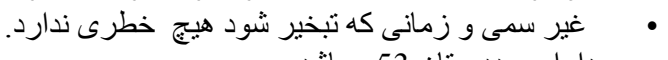

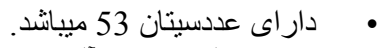

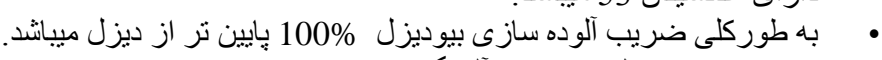

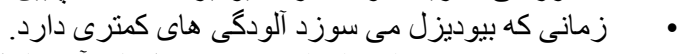

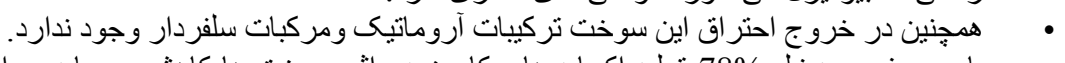

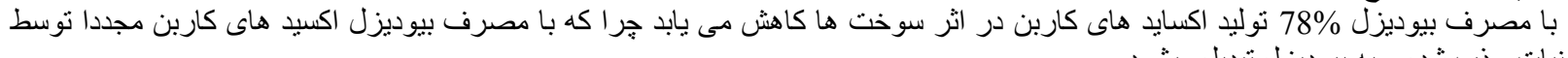

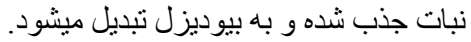

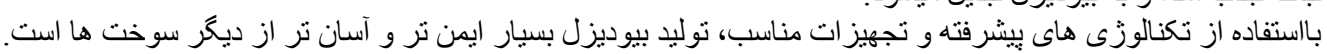

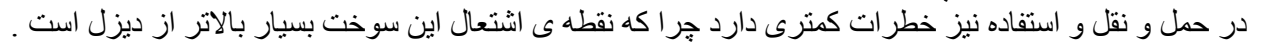




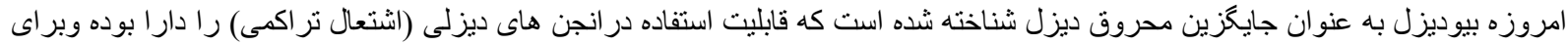

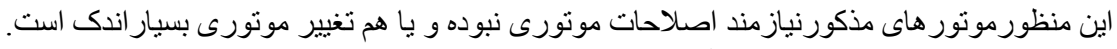

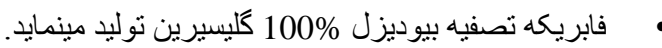

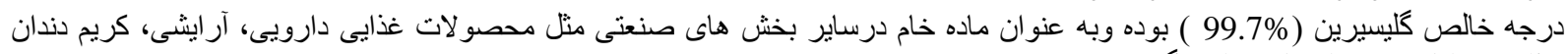

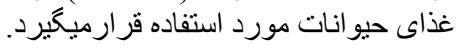

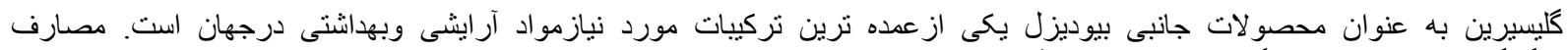

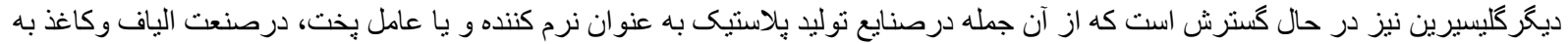

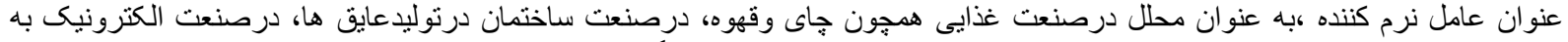

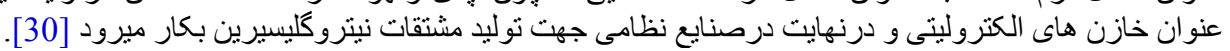
برتزى و نواقص بيوديزل

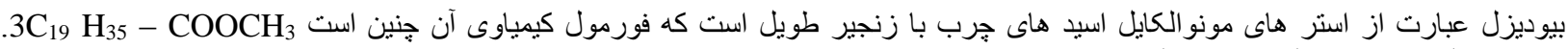
خصوصيات بيوديزل نسبت به ديزل نفتى در ذيل ذيل ذكر شده اند: هاى

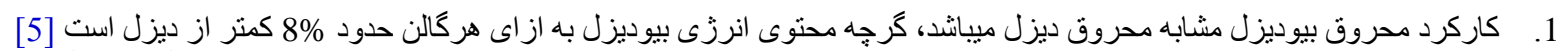
2.

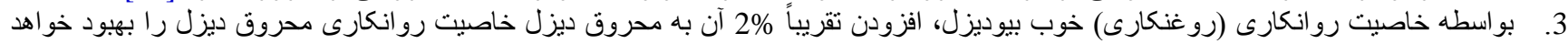

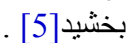

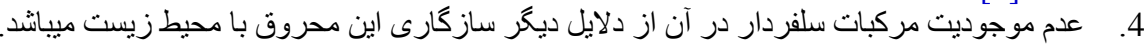
5.

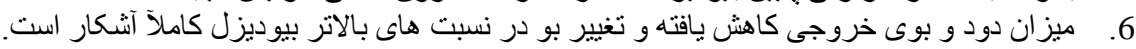
7.

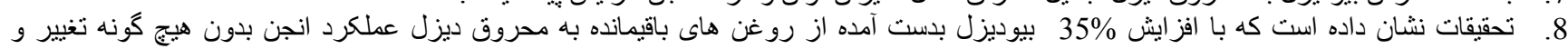

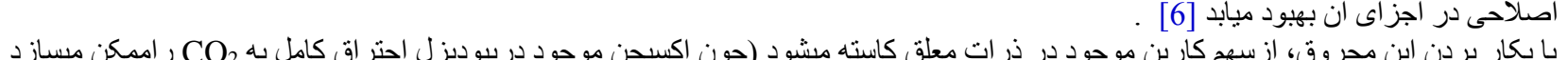

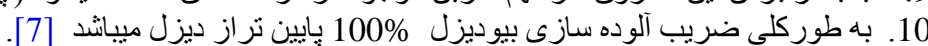

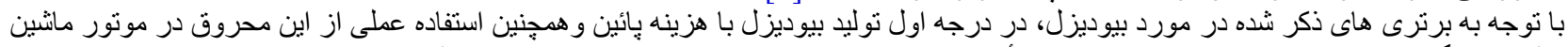

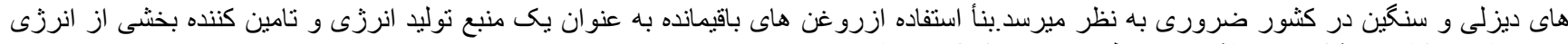
مورد نباز ، به كاهش مشكلات بهداشتى ومحيط زيستى نيز نيز كمى مينمايد.

نتيجه كيرى و بيشنهادات .12

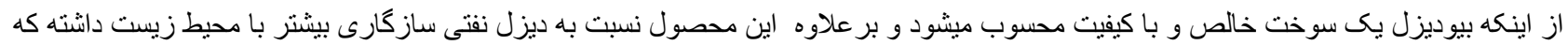

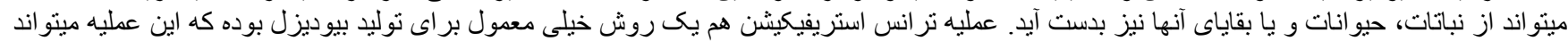

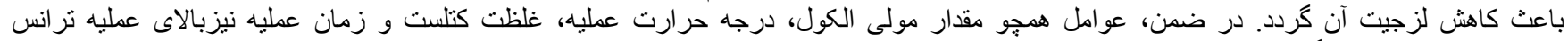

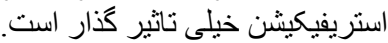

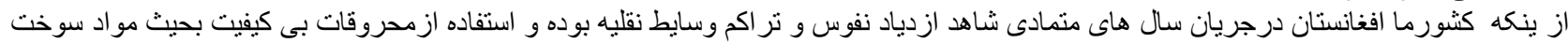

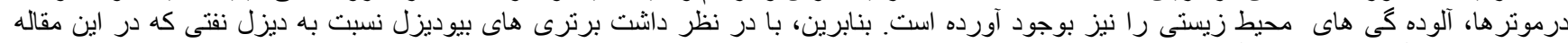

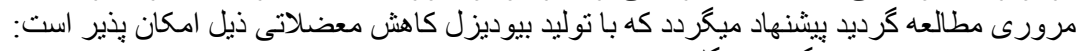

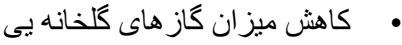

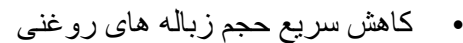

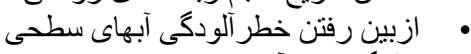

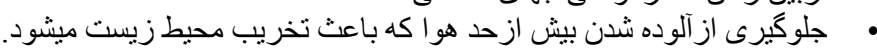

\section{References}

[1] A. N. Phan and T. M. Phan, "Biodiesel production from waste cooking oils," Fuel, vol. 87, pp. 3490-3496, 2008. Available at: https://doi.org/10.1016/j.fuel.2008.07.008.

[2] B. Najafi, V. Pirouzpanah, G. Najafi, T. Yusaf, and B. Ghobadian, "Experimental investigation of performance and emission parameters of a small diesel engine using CNG and biodiesel," SAE Technical Paper2007.

[3] K. Abed, A. K. El Morsi, M. M. Sayed, A. El Shaib, and M. Gad, "Effect of waste cooking-oil biodiesel on performance and exhaust emissions of a diesel engine," Egyptian Journal of Petroleum, vol. 27, pp. 985-989, 2018.

[4] H. U. Cho and J. M. Park, "Biodiesel production by various oleaginous microorganisms from organic wastes," Bioresource Technology, vol. 256, pp. 502-508, 2018. Available at: https://doi.org/10.1016/j.biortech.2018.02.010.

[5] https://blog.ucsusa.org/jeremy-martin/all-about-biodiesel., n.d.

[6] A. G.-R. F. E. S. Carlos and F. A. Guerrero, Biodiesel production from waste cooking oil: InTech, 2011.

[7] G. L. Maddikeri, A. B. Pandit, and P. R. Gogate, "Intensification approaches for biodiesel synthesis from waste cooking oil: A review," Industrial \& Engineering Chemistry Research, vol. 51, pp. 14610-14628, 2012. Available at: https://doi.org/10.1021/ie301675j.

[8] D. McLean and M. Kates, "Biodiesel production from waste cooking oil: 2. Economic assessment and sensitivity analysis," Bioresource Technology, vol. 90, pp. 229-240, 2003. Available at: https://doi.org/10.1016/s0960-8524(03)00150-0.

[9] M.R. and C. Ltd, "Biodiesel: 2018 world market outlook and forecast up to 2027. Retrieved from https://mcgroup.co.uk/researches/biodiesel," 2018. 
[10] V. Cordero-Ravelo and J. Schallenberg-Rodriguez, "Biodiesel production as a solution to waste cooking oil (WCO) disposal. Will any type of WCO do for a transesterification process? A quality assessment," Journal of Environmental Management, vol. 228, pp. 117-129, 2018. Available at: https://doi.org/10.1016/j.jenvman.2018.08.106.

[11] M. Veillette, M. Chamoumi, J. Nikiema, N. Faucheux, and M. Heitz, "Production of biodiesel from microalgae," Advances in Chemical Engineering, vol. 10, pp. 245-260, 2012.

[12] A. Talebian-Kiakalaieh, N. A. S. Amin, and H. Mazaheri, "A review on novel processes of biodiesel production from waste cooking oil," Applied Energy, vol. 104, pp. 683-710, 2013. Available at: https://doi.org/10.1016/j.apenergy.2012.11.061.

[13] Y. Wang, S. Ou, P. Liu, F. Xue, and S. Tang, "Comparison of two different processes to synthesize biodiesel by waste cooking oil," Journal of Molecular Catalysis A: Chemical, vol. 252, pp. 107-112, 2006. Available at: https://doi.org/10.1016/j.molcata.2006.02.047.

[14] M. Allen and G. Prateepchaikul, "The modelling of the biodiesel reaction," Consulted el, 2000.

[15] H. J. Kim, B. S. Kang, M. J. Kim, Y. M. Park, D. K. Kim, J. S. Lee, and K. Y. Lee, "Transesterification of vegetable oil to biodiesel using heterogeneous base catalyst," Catalysis Today, vol. 93-95, pp. 315-320, 2004.

[16] Y. Watanabe, Y. Shimada, A. Sugihara, and Y. Tominaga, "Enzymatic conversion of waste edible oil to biodiesel fuel in a fixed-bed bioreactor," Journal of the American Oil Chemists' Society, vol. 78, pp. 703-707, 2001. Available at: https://doi.org/10.1007/s11746-001-0329-5.

[17] Y. Zhang, M. A. Dubé, D. D. McLean, and M. Kates, "Biodiesel production from waste cooking oil:1. Process design and technological assessment, Bioresour," Technology, vol. 89, pp. 1-16, 2003. Available at: https://doi.org/10.1016/s09608524(03)00040-3.

[18] S. K. Karmee, R. D. Patria, and C. S. K. Lin, "Techno-economic evaluation of biodiesel production from waste cooking oil—a case study of Hong Kong," International Journal of Molecular Sciences, vol. 16, pp. 4362-4371, 2015. Available at: https://doi.org/10.3390/ijms16034362.

[19] M. Mathiyazhagan and A. Ganapathi, "Factors affecting biodiesel production," Research in Plant Biology, vol. 1, pp. 1-5, 2011. Available at: https://doi.org/10.1002/9781119729969.ch1.

[20] A. E. Atabani, A. S. Silitonga, I. A. Badruddin, T. Mahlia, H. Masjuki, and S. Mekhilef, "A comprehensive review on biodiesel as an alternative energy resource and its characteristics," Renewable and Sustainable Energy Reviews, vol. 16, pp. 2070-2093, 2012. Available at: https://doi.org/10.1016/j.rser.2012.01.003.

[21] J. M. Encinar, J. F. Gonzalez, and A. Rodríguez-Reinares, "Biodiesel from used frying oil. Variables affecting the yields and characteristics of the biodiesel," Industrial \& Engineering Chemistry Research, vol. 44, pp. 5491-5499, 2005. Available at: https://doi.org/10.1021/ie040214f.

[22] G. Z. Ares, "Study of factors influcencing the quality and yield of biodiesel produced by transesterification of vegetable oils," 2009.

[23] I. A. Musa, "The effects of alcohol to oil molar ratios and the type of alcohol on biodiesel production using transesterification process," Egyptian Journal of Petroleum, vol. 25, pp. 21-31, 2016. Available at: https://doi.org/10.1016/j.ejpe.2015.06.007.

[24] Y. Wong and S. Devi, "Biodiesel production from used cooking oil," Oriental Journal of Chemistry, vol. 30, pp. 521-528, 2014. Available at: https://doi.org/10.13005/ojc/300216.

[25] R. B. Istiningrum, T. Aprianto, and F. L. U. Pamungkas, "Effect of reaction temperature on biodiesel production from waste cooking oil using lipase as biocatalyst," presented at the AIP Conference Proceedings. AIP Publishing LLC, 2017.

[26] B. Fu, L. Gao, L. Niu, R. Wei, and G. Xiao, "Biodiesel from waste cooking oil via heterogeneous superacid catalyst SO42-/ZrO2," Energy \& Fuels, vol. 23, pp. 569-572, 2009. Available at: https://doi.org/10.1021/ef800751z.

[27] D. Zhang, X. Zhang, Y. Li, S. Wang, X. Wang, and Z. Jiang, "Materials science \& engineering incorporation of Ce3+ ions into dodecatungstophosphoric acid for the production of biodiesel from waste cooking oil," Materials Science and Engineering: C, vol. 92, pp. 922-931, 2018. Available at: https://doi.org/10.1016/j.msec.2018.07.047.

[28] X. Meng, G. Chen, and Y. Wang, "Biodiesel production from waste cooking oil via alkali catalyst and its engine test," Fuel Processing Technology, vol. 89, pp. 851-857, 2008. Available at: https://doi.org/10.1016/j.fuproc.2008.02.006.

[29] O. Samuel, M. Waheed, B. O. Bolaji, and O. Dairo, "Production of biodiesel from Nigeria restaurant waste cooking oil using blender," International Journal of Renewable Energy Research, vol. 3, pp. 996-979, 2013.

[30] N. Mansir, S. H. Teo, I. Rabiu, and Y. H. Taufiq-Yap, "Effective biodiesel synthesis from waste cooking oil and biomass residue solid green catalyst," Chemical Engineering Journal, vol. 347, pp. 137-144, 2018. Available at: https://doi.org/10.1016/j.cej.2018.04.034.

[31] D. Qarizada, E. Mohammadian, A. B. Alias, H. A. Rahimi, S. Binti, and M. Yusuf, "Effect of temperature on bio-oil fractions of palm kernel shell thermal distillation," International Journal of Innovative Research and Advanced Studies, vol. 1, pp. 3439, 2018. 